\title{
An Improved PSO-Based MPPT Control Strategy for Photovoltaic Systems
}

\author{
M. Abdulkadir, A. H. M. Yatim, and S. T. Yusuf \\ Department of Electrical Power Engineering, Faculty of Electrical Engineering, Universiti Teknologi Malaysia, \\ 81310 Skudai, Johor Bahru, Malaysia
}

Correspondence should be addressed to M. Abdulkadir; amusa2@live.utm.my

Received 1 March 2014; Accepted 28 May 2014; Published 16 June 2014

Academic Editor: Cooper H. Langford

Copyright (C) 2014 M. Abdulkadir et al. This is an open access article distributed under the Creative Commons Attribution License, which permits unrestricted use, distribution, and reproduction in any medium, provided the original work is properly cited.

This paper presents a control strategy proposed for power maximizing which is a critical mechanism to ensure power track is maximized. Many tracking algorithms have been proposed for this purpose. One of the more commonly used techniques is the incremental conductance method. In this paper, an improved particle swarm optimization- (IPSO-) based MPPT technique for photovoltaic system operating under varying environmental conditions is proposed. The approach of linearly decreasing scheme for weighting factor and cognitive and social parameter is modified. The proposed control scheme can overcome deficiency and accelerate convergence of the IPSO-based MPPT algorithm. The approach is not only capable of tracking the maximum power point under uniform insolation state, but also able to find the maximum power point under fast changing nonuniform insolation conditions. The photovoltaic systematic process with control schemes is created using MATLAB Simulink to verify the effectiveness with several simulations being carried out and then compared with the conventional incremental conductance technique. Lastly, the effectiveness of the intended techniques is proven using real data obtained form previous literature. With the change in insolation and temperature portrait, it produces exceptional MPPT maximization. This shows that optimum performance is achieved using the intended method compared to the typical method.

\section{Introduction}

Energy is indispensable to human life. Energy is not only a measurement for economic and social improvement but also a fundamental human necessity. Many countries are trying to find means to solve energy problems such as depending on energy importation, minimization of environmental pollution, global warming, increasing cost of energy, and energy inefficiency $[1,2]$.

Photovoltaic (PV) system has gained wide popularity in the past decade as one of the renewable energy sources due to the possibility of depletion of conventional energy sources and its high cost as well as its negative effects on the environment. One essential fundamental of all PV is the efficacy of its maximum power point tracking. The aspect has drawn immense enthusiasm from photovoltaic researchers and industry experts being the most economical means to enhance above all the photovoltaic system efficacy. Maximum power point tracking is primarily an operating point cocoordinating between the photovoltaic module and the DC-DC converter. Nonetheless, maximum power point tracking is not simple and easy to track because of the nonlinear $I-V$ characteristics of the photovoltaic curve and the effect of the changing weather situations (especially radiation and temperature); tracking the accurate maximum power point (MPP) has been always an intricate issue. The tracking eventually is further sophisticated when all photovoltaic modules do not experience constant radiation.

For the past decades, many MPPT algorithms have been proposed, in which many centered around obtaining optimum maximum power point [2-6]. Among the renowned power maximizing methods are Perturb and Observe (P\&O) and/or hill climbing and incremental conductance (IC) [7-9]. These techniques, nonetheless, fail to track maximum power point when the insolation level is not consistent for all PV solar cells or the panel is partially shaded. $\mathrm{P} \& \mathrm{O}$ technique frequently leads to wrongful conclusion, oscillation around the maximum power point, and it generally needs to link one or many modifications for general usage. Incremental 
conductance techniques overcome these shortfalls of Perturb and Observe techniques but need relatively elaborate detection devices, and the choice of the step and threshold is also distressing [10].

Recently, numerous researchers have presented intelligent MPPT methods [5-11] for photovoltaic module arrays, both to track MPPs accurately and to improve the dynamic and steady-state tracking performance. However, these methods are applicable only to MPPT in photovoltaic $c$ module arrays without shading. Nevertheless, the appearance of multipeak output curves because of partial module shading in photovoltaic module arrays is common. Therefore, the development of an algorithm for accurately tracking the true MPPs of complex and nonlinear output curves is crucial. Reference [12] presented a MPP tracker based on particle swarm optimization (PSO) for photovoltaic module arrays. Although this tracker was capable of tracking global MPPs of multipeak characteristic curves because fixed values were adopted for weighing within the algorithm, the tracking performance lacked robustness, causing low success rates when tracking global MPPs. Even though the MPPs were tracked successfully, the dynamic response speed was low. Therefore, this study used PSO and added improvements, preventing it from being trapped in local MPPs (i.e., searching only local MPPs) and enabling it to track global MPPs quickly and consistently on the multipeak characteristic curves of photovoltaic module arrays.

An alternative approach is to employ evolutionary algorithm (EA) techniques. Due to its ability to handle nonlinear objective functions [13], EA is envisaged to be very effective to deal with MPPT problem. Among the EA techniques, particle swarm optimization (PSO) is highly potential due to its simple structure, easy implementation, and fast computation capability [13]. Since PSO is based on search optimization, in principle, it should be able to locate the MPP for any type of $P$ $V$ curve regardless of environmental variations. It can be used to track the MPP of PV system as the search space of the PSO is reduced, and, hence, the time required for convergence can be greatly decreased.

Interestingly, one important feature of the PSO which is ignored by researchers $[5,6,13,14]$ is the searching speed through adaptive learning factors and inertia weight. Linear decreases in line with increasing iteration numbers were adopted in this study for the weighting of the PSO formulas. The physical meaning of this modified weighting formula is that greater step sizes are used to increase the particle search velocity during the initial search because the distance to the global optimum is relatively large. This prevents an excessively small step size from making local optimum traps unavoidable.

However, $w$ decreases gradually as the number of iterations increases. As the particles approach the MPP, this decrease in $w$ causes the steps in the particle movements to diminish, enabling the particles to track the MPP more accurately. In PSO equation, the first term $w(t) v_{i}(t)$ is exploited to maintain the same direction in which the particle was moving pristinely; thereby controlling the converging demeanor of the particle swarm optimization. In order to expedite converging, the inertia weight will be culled such that the effect of $v_{i}(t)$ of the algorithm diminishes during the operation. Therefore, the value of $w$ decrementing with time is desirable. To get refined solutions, a very popular option is to set the inertia weight initially to a bigger value for better exploration and then reduce it gradually. Likewise, the cognitive and social parameter can also be modified as it affects the search ability of PSO. Therefore, power loss as a result of the oscillation is eradicated and the efficiency of the system increases. The theoretical analysis and simulation results presented illustrate the good performances of the proposed control schemes.

\section{System Overview}

Photovoltaic system models have long time been an origin for the description of photovoltaic nature for researchers and professionals alike. The utmost ordinary model used to foresee energy generation in photovoltaic cell model is the single diode circuit $[3,15]$. An ideal photovoltaic cell is comprised of a single diode connected in parallel with a light current source as depicted in Figure 1. A complete PV system simulation should fulfill the following criteria: (a) it should be simple and fast but able to correctively predict the $I-V$ and $P-V$ characteristic curves, including special conditions such as partial shading; and (b) it should be an overall tool which can evolve and ratify a photovoltaic system design allencompassing the power converter and the MPPT control as shown in Figure $2[3,15,16]$.

\section{Basic of Incremental Conductance and PSO}

In this work, the performance of the proposed CS will be evaluated in comparison to Perturb and Observation (P\&O) and Particle Swarm Optimization (PSO) MPPT. To facilitate discussion that shall follow, brief overviews of both methods are presented here.

3.1. Incremental Conductance (IC). The idea behind incremental conductance is to increasingly contrast the ratio of the derivative of conductance with the instantaneous conductance $[1,17]$. It is derived from the fact that, at MPP, the derivative of power with respect to voltage $(d P / d V)$ is, in fact, zero; that is,

$$
\frac{\partial P_{\mathrm{PV}}}{\partial V_{\mathrm{PV}}}=\frac{\partial\left(V_{\mathrm{PV}} * I_{\mathrm{PV}}\right)}{\partial V_{\mathrm{PV}}}=V_{\mathrm{PV}} * \frac{\partial I_{\mathrm{PV}}}{\partial V_{\mathrm{PV}}}+I_{\mathrm{PV}}=0,
$$

where the change in photovoltaic current is $\partial I_{\mathrm{PV}}$, the change in photovoltaic voltage is $\partial V_{\mathrm{PV}}$, and $\partial P_{\mathrm{PV}}$ is the change in photovoltaic power, respectively.

Equation (1) can be reconstructed as follows:

$$
\frac{\partial I_{\mathrm{PV}}}{\partial V_{\mathrm{PV}}}=-\frac{I_{\mathrm{PV}}}{V_{\mathrm{PV}}} \approx \frac{\Delta I}{\Delta V}
$$

where photovoltaic current and voltage $\Delta I$ and $\Delta V$ are the increments, respectively. 


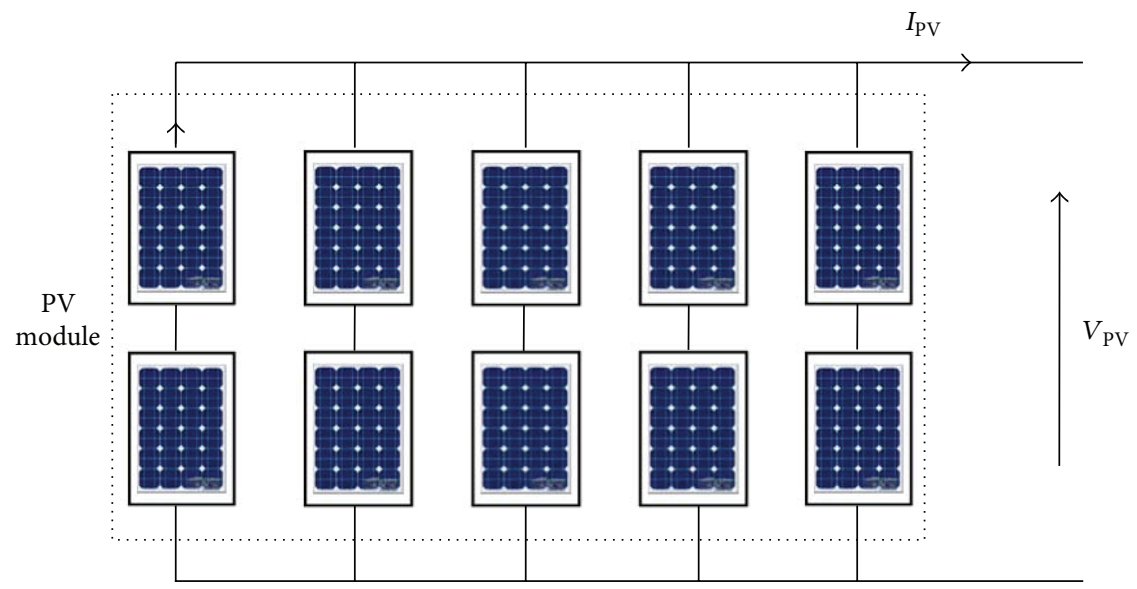

FIGURE 1: Photovoltaic System.

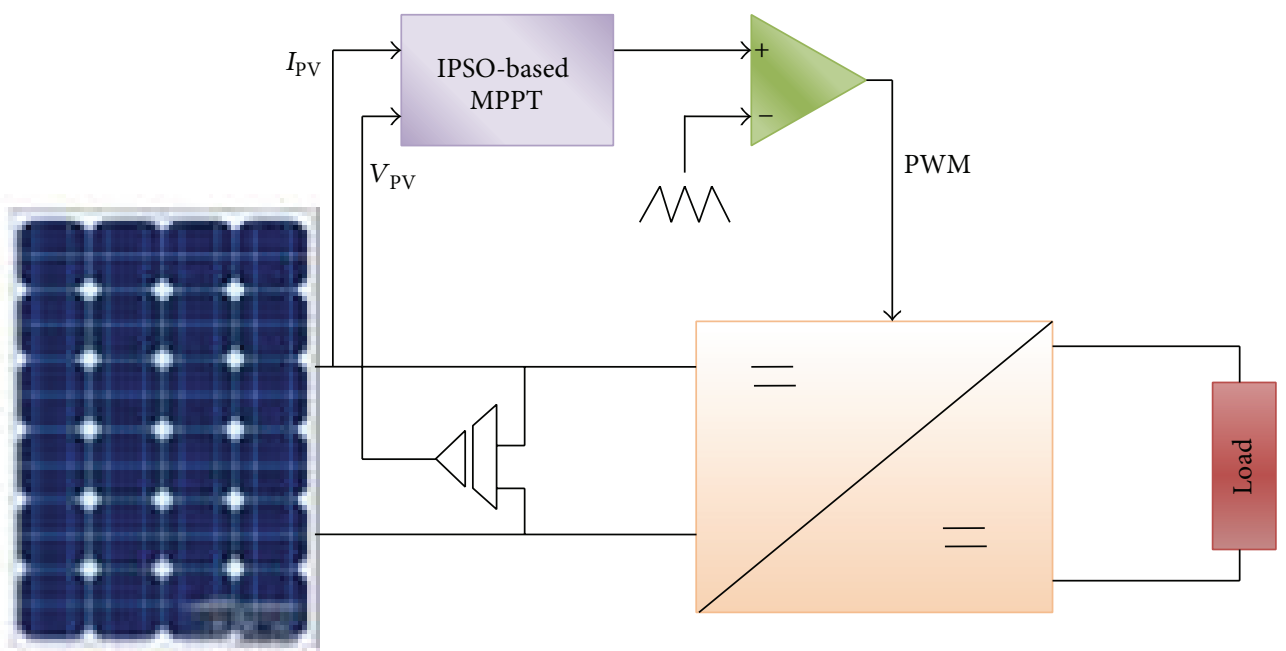

FIGURE 2: Configuration of the proposed PV system.

The key rules for incremental conductance can hence be derived from the $P-V$ characteristics and written as follows $[18,19]$ :

$$
\begin{array}{ll}
\frac{\partial P_{\mathrm{PV}}}{\partial V_{\mathrm{PV}}}>0 & \text { if } \frac{I_{\mathrm{PV}}}{V_{\mathrm{PV}}}>-\frac{\partial I_{\mathrm{PV}}}{\partial V_{\mathrm{PV}}}, \text { on the left of MPP; } \\
\frac{\partial P_{\mathrm{PV}}}{\partial V_{\mathrm{PV}}}=0 & \text { if } \frac{I_{\mathrm{PV}}}{V_{\mathrm{PV}}}=-\frac{\partial I_{\mathrm{PV}}}{\partial V_{\mathrm{PV}}}, \text { at the MPP; } \\
\frac{\partial P_{\mathrm{PV}}}{\partial V_{\mathrm{PV}}}<0 & \text { if } \frac{I_{\mathrm{PV}}}{V_{\mathrm{PV}}}<-\frac{\partial I_{\mathrm{PV}}}{\partial V_{\mathrm{PV}}}, \text { on the right of MPP, }
\end{array}
$$

where MPP refers to maximum power point.

Using the rules in (3), it can be observed that the reference signal is based on voltage $V^{*}$. As the rules in (3) are derived using $P-V$ curve, the current cannot be used as the final output; rather, $P-I$ characteristics curve is utilized. The clear flow chart of this technique is reported in [1]. The following are the modification in respect of the standard incremental conductance algorithm.
3.2. Particle Swarm Optimization (PSO). Particle swarm optimization (PSO) is a stochastic, population-based EA search method, modeled after the behavior of bird flocks [13]. The PSO algorithm maintains a swarm of individuals (called particles), where each particle represents a candidate solution. Particles follow a simple behavior: emulate the success of neighboring particles and its own achieved successes. The position of a particle is therefore influenced by the best particle in a neighborhood, $p_{\text {best }}$, as well as the best solution found by all the particles in the entire population, $g_{\text {best }}$. The particle position, $x_{i}$, is adjusted using [13]

$$
x_{i}^{t+1}=x_{i}^{t}+v_{i}^{t+1}
$$

where the velocity component, $v_{i}$, represents the step size.

The velocity is calculated by

$$
\begin{array}{r}
v_{i}^{t+1}=w v_{i}^{t}+c_{1} r_{1} \cdot\left(p_{\text {best }}, i-x_{i}^{t}\right)+c_{2} r_{2} \cdot\left(g_{\text {best }}-x_{i}^{t}\right), \\
i=1,2, \ldots, N,
\end{array}
$$




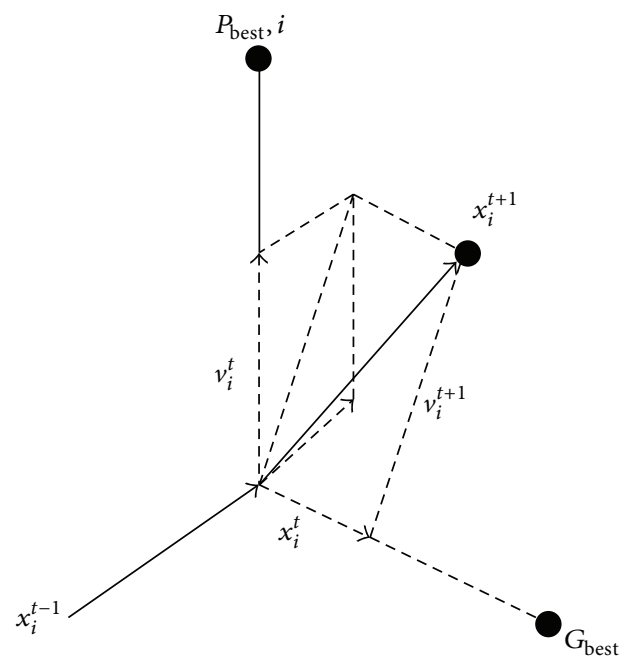

FIGURE 3: Movement of particles in the optimization process.

where $x_{i}$ denote the particle position for $i$; the velocity of the particle at $i$ is represented by $v_{i}$; the number of iteration is denoted by $t$; the inertia weight is represented by $w ; r_{1}$ and $r_{2}$ are uniformly distributed random variables within $[0,1]$; and the cognitive and social coefficient are, respectively, denoted by $c_{1}, c_{2}$ [14]. The best position for the storage of the $i$ th particle that has been found so far is denoted by variable $p_{\text {best }}, i$ and the storage of the best position of all the particles is represented by $g_{\text {best }}$. Figure 3 depicts the movement of particle in the optimization process.

3.3. The Weakness of Conventional Particle Swarm Optimization-Based MPPT Techniques. Conventional PSO is fast and accurate when searching for the output characteristic curves of PV module arrays with single peak values. However, when some modules are shaded, weights in conventional PSO must be readjusted appropriately based on various multipeak curve characteristics. If this is not performed, excessively high or low weights result in tracking failure. Thus, conventional PSO-based MPPT must be modified when some of the modules in a photovoltaic module array are shaded.

\section{IPSO-Based MPPT Technique}

For the IPSO-based MPPT system designed, the position of the particle is designated as the duty cycle of the power converter, while the fitness value evaluation function was chosen as the produced power $P_{\mathrm{PV}}$ for the entire photovoltaic system. In the proposed method overview, more accurate MPP tracking is achieved despite the complex shading conditions with the smaller particle number and where larger number of particles results in lengthy computation time. Therefore, for good tracking speed and accuracy to be ensured, a tradeoff should be made. According to some research, at most there exist $m$ MPPs in the $P-V$ curve for photovoltaic modules which consist of $m$ series connected photovoltaic cells [12]. For initialization step of the particle swarm optimization, particles could be established in the random range or be placed on stationary position. Mostly, it makes more sense to initialize the particles around it if there is data available regarding the position of the global maximum power point in the search range. In [12], the authors state that the minimum displacement between successive peaks is nearly $80 \%$ of $V_{\mathrm{OC}}$ and also the peaks on the $P-V$ curve occur nearly at multiples of $80 \%$ of the module open voltage $V_{\mathrm{OC}}$. Thus, the number of particle $N$ is selected in the photovoltaic system as the number of the series connected cells. The search spaces of the particles that cover [ $\left[\begin{array}{ll}0 & 1\end{array}\right]$ are initialized on definite point. 0 and 1 are the duty cycle minimum and maximum value of the dc-dc converter used, respectively.

The objective of this IPSO-based MPPT method was to extract the maximum power $P_{\mathrm{PV}}$ of the photovoltaic. To evaluate the fitness value which is the generated power, after the controller output, the pulse width modulation acts in line to the particle position $i$ that denote the duty cycle state, and the photovoltaic voltage $V_{\mathrm{PV}}$ and current $I_{\mathrm{PV}}$ can be measured. To calculate the fitness value $P_{\mathrm{PV}}$ of particle $i$, these values can then be used. However, to obtain the right samples time, it should be noted that power converter's settling time has to be lesser than the evaluations time interspaces between subsequent particles.

To address these problems, linear decreases with increasing iteration numbers were adopted in this study for the weighting of the PSO formulas. The physical meaning of this modified weighting formula is that greater step sizes are used to increase the particle search velocity during the initial search because the distance to the global optimum is relatively large. This prevents an excessively small step size from making local optimum traps unavoidable. However, $w$ decreases gradually as the number of iterations increases. Because the particles are now approaching the MPP, these decreases in $w$ cause the steps in the particle movements to shrink, enabling the particles to track the MPP more accurately.

In (5), in order to maintain the particle accelerating in the same direction it was originally moving, the first term $w(t) v_{i}(t)$ is therefore utilized, where the converging demeanor of the particle swarm optimization is controlled. The inertia weight will be chosen in order to accelerate convergence, such that the effect of $v_{i}(t)$ diminished through the process of the algorithm. Hence, the choice of a decreasing value of $w$ with time is considered. To get refined solutions, a typical option is to set the inertia weight initially to a bigger value and slowly reduce it for better exploration. For this reason, here, the term $w$ was used as linearly decreasing scheme, as illustrated as follows:

$$
w(t)=w_{\max }-\frac{t}{t_{\max }}\left(w_{\max }-w_{\min }\right) .
$$

In (6), the minimum and maximum bounds of $w$ were denoted by $w_{\min }$ and $w_{\max }$, while the maximum allowed number of iterations are denoted by $t_{\max }$. Likewise, the social and cognitive terms can be remodelled. In (5), the search ability of particle swarm optimization can be affected by the values of $c_{1}$ and $c_{2}$ by changing the particle direction. Selecting $c_{1}>c_{2}$ sampling with respect to the bearing of $p_{\text {best }}, i$ would be biased, while selecting $c_{1}<c_{2}$ in the reverse case, sampling in respect to the bearing of $g_{\text {best }}$ will be preferred. 


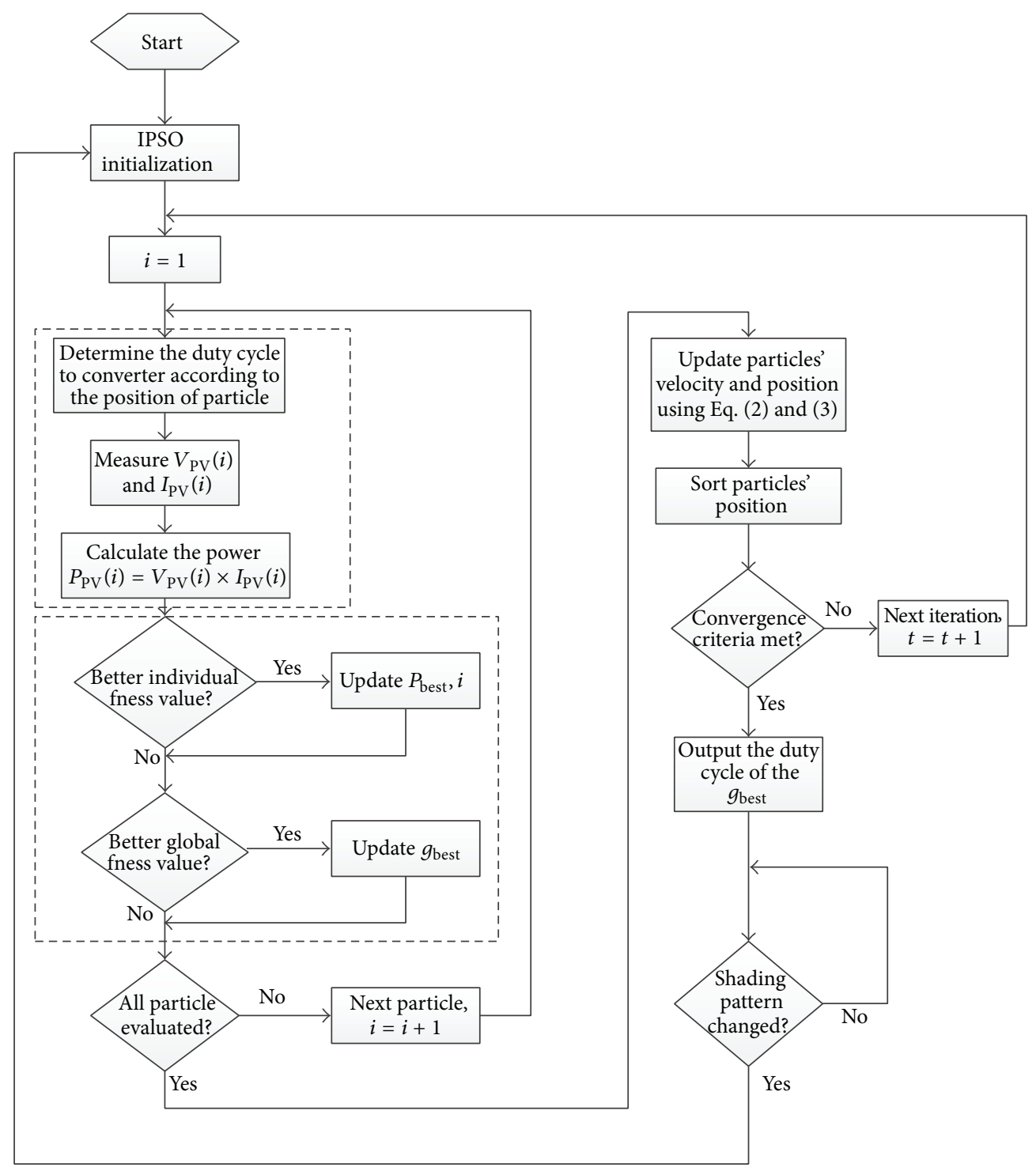

FIGURE 4: Flowchart of the proposed IPSO-based MPPT algorithm.

For these reasons, these two terms are defined as continuously increasing and continuously decreasing functions, as in (7) and (8) respectively:

$$
\begin{aligned}
& c_{1}(t)=c_{1, \max }-\frac{t}{t_{\max }}\left(c_{1, \max }-c_{1, \min }\right), \\
& c_{2}(t)=c_{2, \max }-\frac{t}{t_{\max }}\left(c_{2, \max }-c_{2, \min }\right) .
\end{aligned}
$$

Two convergence criteria are employed in this study. The proposed IPSO-based MPPT method will halt and yield the $g_{\text {best }}$ solution, if the maximum number of iterations is attained or if all the particles velocities become smaller than a threshold.

Basically, particle swarm optimization algorithms are utilized to address optimization difficulty that the optimum result is time invariant. However, in this case, the fitness value which is the global maximum power point sometimes varies or depends on environmental factors as well as loading states. To search for the new global MPP again in these cases, the particles must be reinitialized. Considering the change in insolation and shading pattern to be detected, here, the following constraint is utilized. In the proposed IPSO-based technique, the particles will be reinitializing whenever the following condition is satisfied as shown in (9); Figure 4 depicts the comprehensive flowchart of the proposed system:

$$
\frac{\left|P_{\mathrm{PV}, \text { new }}-P_{\mathrm{PV} \text {, old }}\right|}{P_{\mathrm{PV} \text {, old }}} \geq \Delta P(\%),
$$

where $P_{\mathrm{PV} \text {, new }}$ is the new photovoltaic power, $P_{\mathrm{PV} \text {, old }}$ is the photovoltaic power at global maximum point of the last operating point, and $\Delta P(\%)$ is set to $10 \%$.

4.1. Effect of Partial Shading. A photovoltaic module is comprised of many photovoltaic cells either connected in series to produce a higher voltage or connected in parallel to increase current. Many photovoltaic cells are therefore connected either in series or in parallel to form a photovoltaic system. 


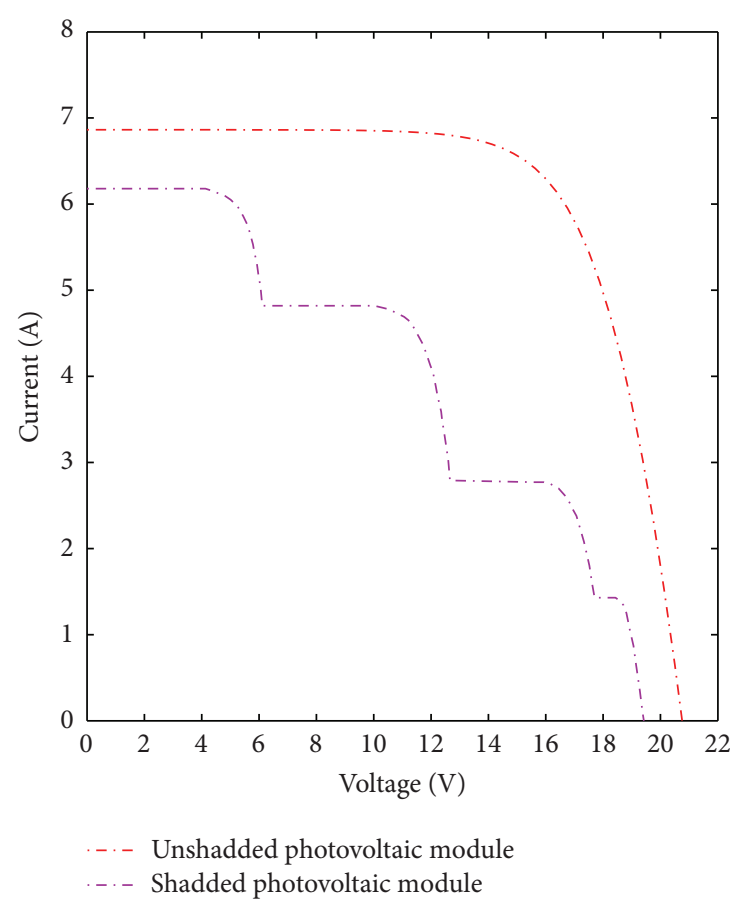

(a)

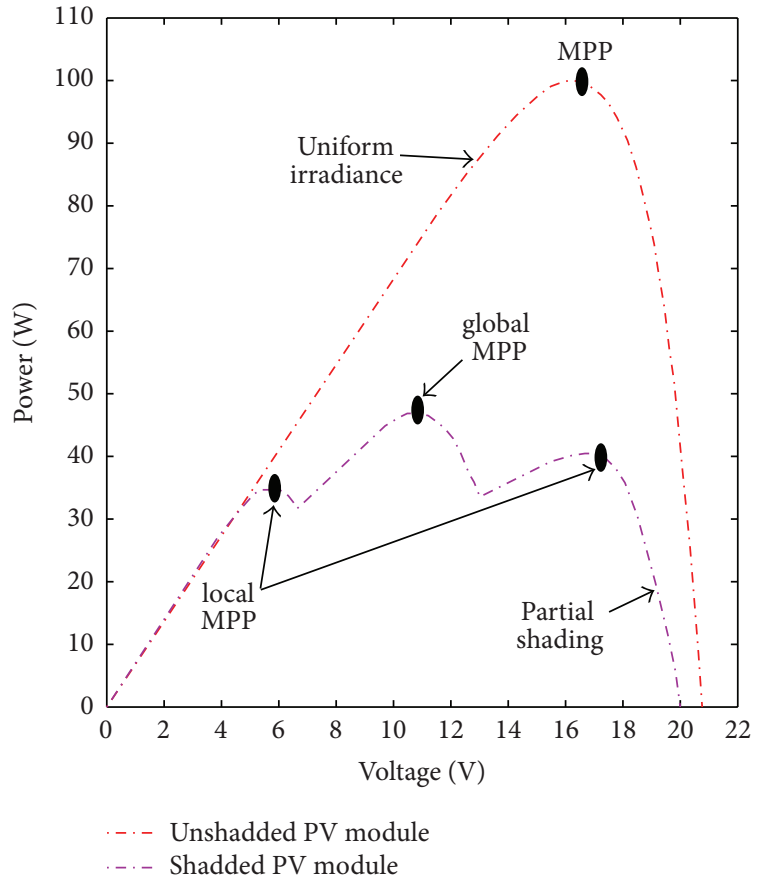

(b)

FIGURE 5: (a) shows $I-V$ characteristic of PV system under shading and nonshading condition and (b) shows the $P-V$ characteristic of PV system under shading and nonshading condition, respectively.

The $P$-V curve of photovoltaic cell would exhibit multiple MPPs under partial shading pattern, because of the bypass diodes as reported in [17-20]. The photovoltaic module characteristics under partial shading pattern connected at module terminal with bypass diodes can be described as bellows. In partial shading pattern, the shaded portion of the cells acts as a load rather than a generator and creates the hot shot and, hence, the bypass diodes of these shaded cells will conduct to avert this bad situation [17]. Multiple peaks in the $P-V$ curve would be obtained since the shaded modules are bypassed. The resulting $I-V$ curves when this system is under different shading conditions are shown in Figure 5(a). Same process can then be used to get the $P$ $V$ characteristic curves as illustrated in Figure 5(b). It can then be noticed from Figure 5(a) that the global MPP could happen, depending on the type of shading pattern in either the below or above voltage range. For this reason, the conventional MPPT algorithms will be very difficult to be applied directly.

\section{Simulation Results and Discussion}

To demonstrate the effectiveness of the proposed IPSO-based MPPT technique, simulations were performed appropriately. The simulation model parameters of the PV module used are shown in Table 1.

In this paper, the simulations were implemented using MATLAB/Simulink model. According to the design principle, the specification frameworks of the complete IPSO-based MPPT algorithm are shown in Table 2. The photovoltaic module characteristic curves are simulated by arbitrary
TABLE 1: Simulation model parameters of ICO-SPC $100 \mathrm{~W}$ photovoltaic module [3].

\begin{tabular}{lc}
\hline Parameter & Value \\
\hline Maximum Power $\left(P_{\max }\right)$ & $100 \mathrm{~W}$ \\
Voltage at $P_{\max }\left(V_{\max }\right)$ & $17.3 \mathrm{~V}$ \\
Current at $P_{\max }\left(I_{\max }\right)$ & $5.79 \mathrm{~A}$ \\
Open circuit Voltage $\left(V_{\mathrm{oc}}\right)$ & $20.76 \mathrm{~V}$ \\
Short circuit Current $\left(I_{\mathrm{sc}}\right)$ & $6.87 \mathrm{~A}$ \\
Number of cells in series $\left(N_{s}\right)$ & 36 \\
Number of cells in parallel $\left(N_{p}\right)$ & 1 \\
\hline
\end{tabular}

TABLE 2: Simulation parameter setting of the IPSO-based MPPT.

\begin{tabular}{lc}
\hline Parameter & Value \\
\hline Number of particles & 3 \\
Minimum duty cycle & 0.02 \\
Maximum duty cycle & 0.98 \\
Sampling time & $0.1 \mathrm{~s}$ \\
Maximum iteration & 3 \\
$W_{\max }$ & 1.0 \\
$W_{\min }$ & 0.1 \\
$C_{1, \min }$ & 1 \\
$C_{1, \max }$ & 1.2 \\
$C_{2, \min }$ & 1 \\
$C_{2, \max }$ & 1.6 \\
\hline
\end{tabular}

setting the irradiation of the series connected photovoltaic cells under the effect of partial shading condition. The 


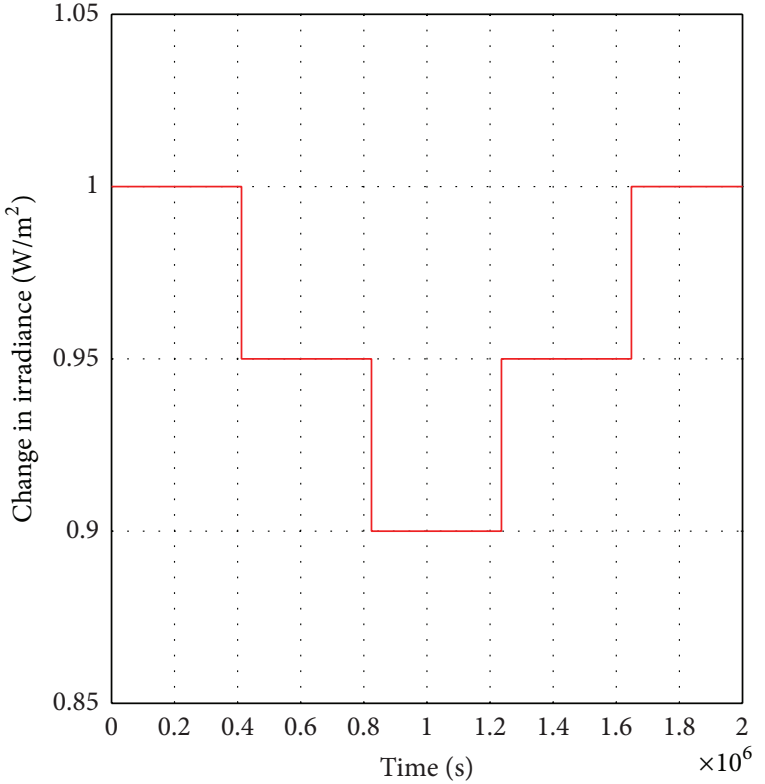

(a)

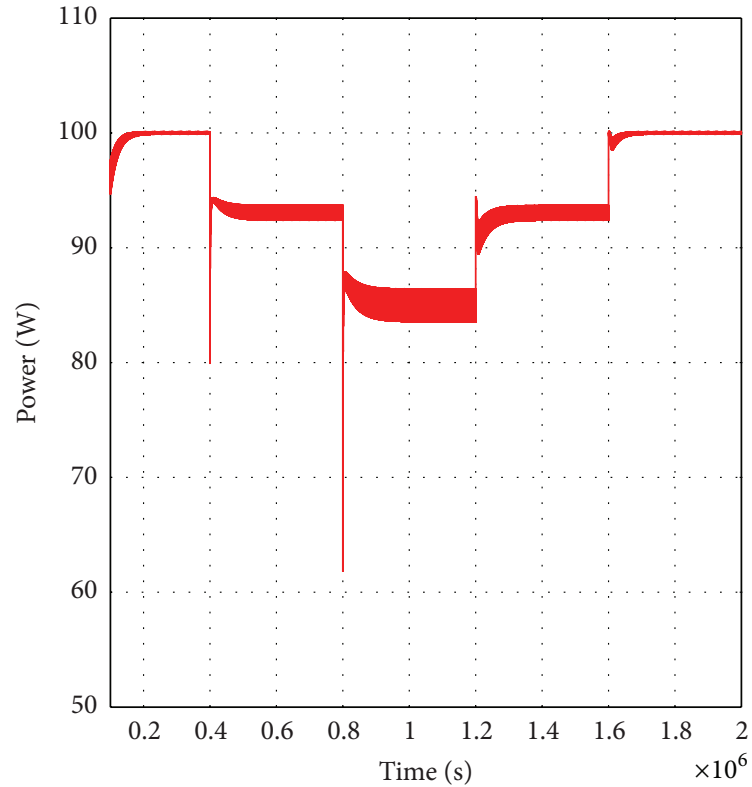

(b)

FIGURE 6: (a) shows varying irradiance level and (b) shows maximum power due to irradiance variation.

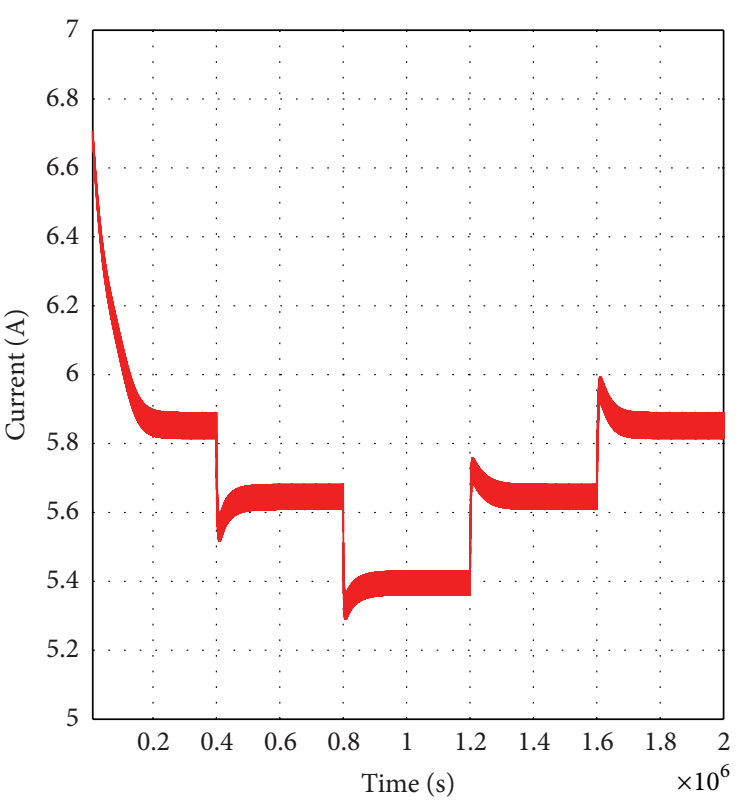

(a)

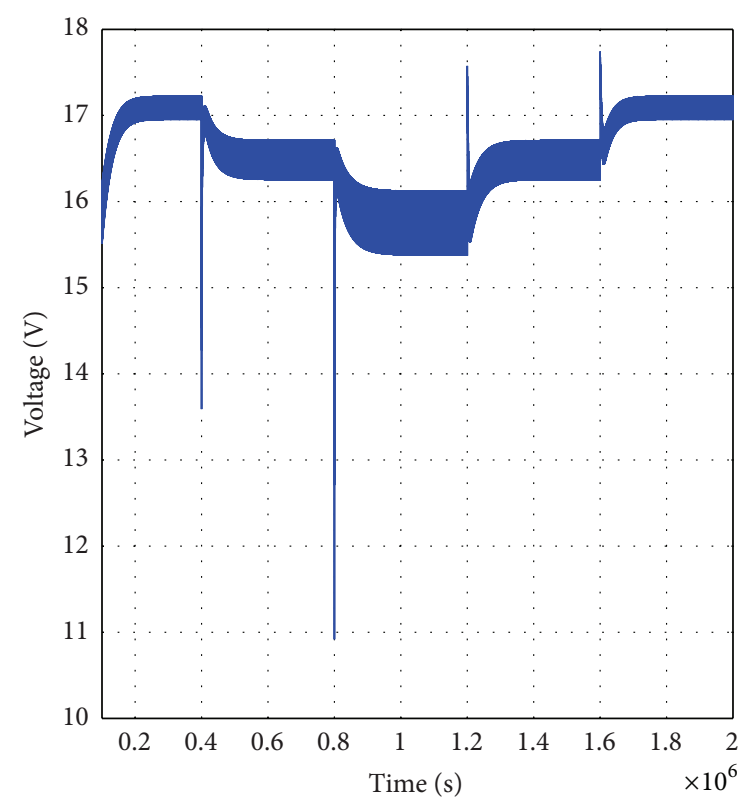

(b)

FIGURE 7: (a) shows the current at maximum power under varying irradiance condition and (b) shows voltage at maximum power under varying irradiance condition.

photovoltaic module temperature is taken to be unchanged at $25^{\circ} \mathrm{C}$ during the simulation. Unshaded photovoltaic modules are thoughtout entirely radiated at $1000 \mathrm{~W} / \mathrm{m}^{2}$. Irradiation on shaded photovoltaic module is thoughtout constant and change from 0 to $1000 \mathrm{~W} / \mathrm{m}^{2}$. For different shading patterns, the IPSO-based MPPT algorithms are tested and verified and the simulation results are presented in Figures 6 and 7, respectively .
From the computed power, the duty cycles are updated according to the proposed algorithm. When there is a change in irradiation from $1000 \mathrm{~W} / \mathrm{m}$ to $950 \mathrm{~W} / \mathrm{m}^{2}$, the duty cycles are recomputed using (4) and (5) for the tracking of new MPP. The search process is continuously changing the duty cycles when irradiation changes from high to low value, that is, $950 \mathrm{~W} / \mathrm{m}$ to $900 \mathrm{~W} / \mathrm{m}^{2}$; new duty cycles are again computed using (4) and (5). At 0.52 sampling period, the steady 


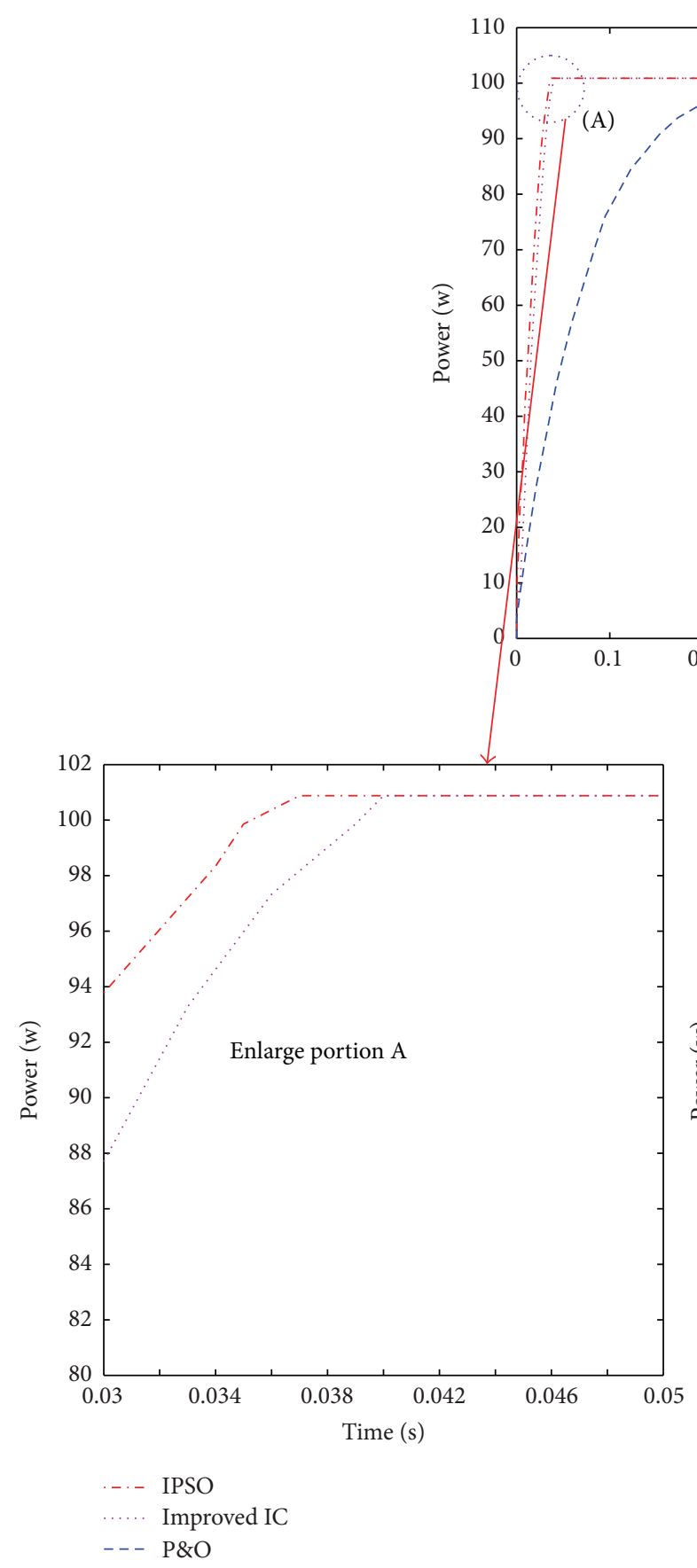

(b)

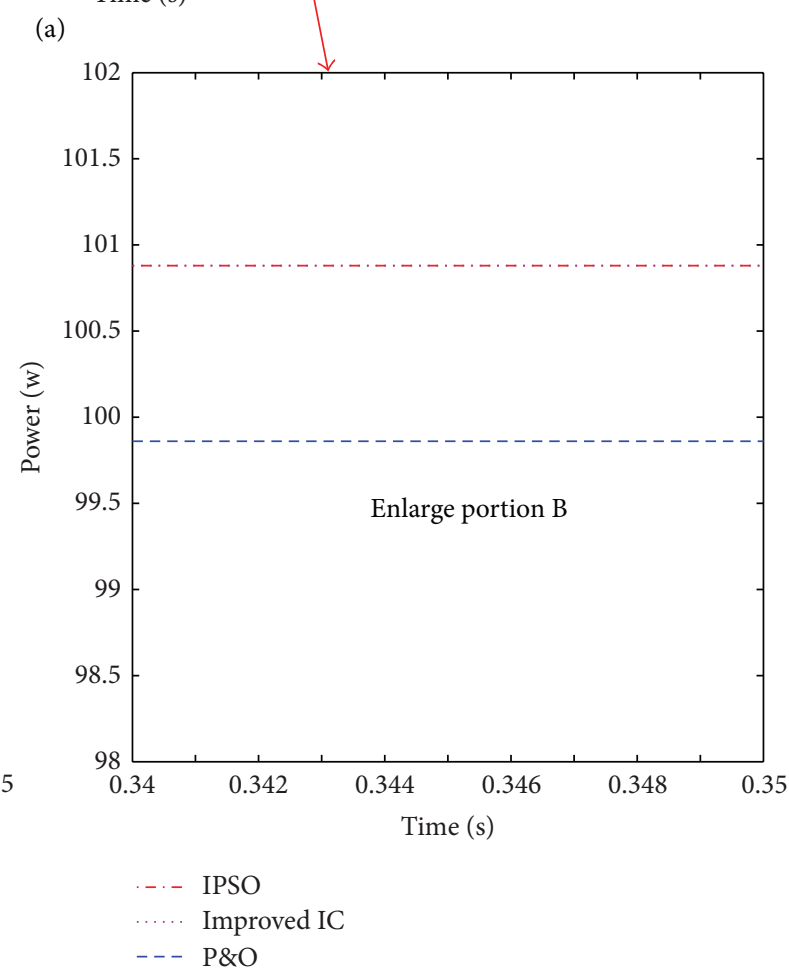

(c)

FIGURE 8: (a) depicts the performance comparison between IPSO, modified IC, and P\&O. (b) Enlarge portion A. (c) Enlarge portion B.

state value is attained. As the duty cycles are reinitialized for every change in irradiation, a fast tracking speed and almost zero steady state oscillations at MPP are attained when compared to IncCond technique which makes IPSO algorithm exceptional.

Figure 8 compares the performance of IPSO, modified IC, and P\&O. For IPSO, variables $c_{1}$ and $c_{2}$ are chosen as shown in Table 2. These values are chosen after extensive simulation trials and thus the IPSO can be regarded as well optimized. The random numbers are generated by
MATLAB rand function. The result indicates that initially IPSO requires a much longer time, that is, approximately $200 \mathrm{~ms}$ to settle at the first MPP. After convergence, both algorithms track the MPP perfectly and remain almost ripple-free at steady state. However, during the power ramp, IPSO sustains a prolong oscillation compared to modified IC. This is due to the smaller step sizes, which forces the IPSO to utilize more samples in order to converge to MPP. This observation is consistent with discussions presented in Section 4. 


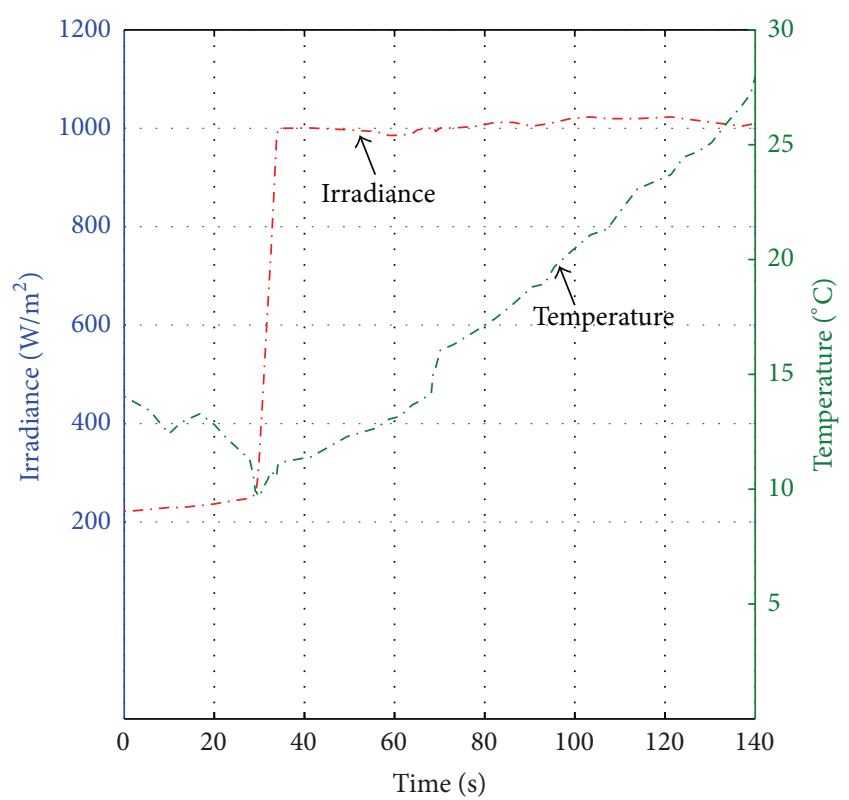

FIGURE 9: Depict a sequence of a rapid irradiation and temperature variation [11].

Throughout the experiment, the temperature is maintained constant at $25^{\circ} \mathrm{C}$. This value is very conservative, considering the PV cell string voltage is $20.76 \mathrm{~V}$. Furthermore, it can be seen that even with a small step-size, $\mathrm{P} \& \mathrm{O}$ oscillate (around the MPP) with an average ripple of $100 \mathrm{~W}$. On the other hand, as modified IC continuously sticks to the MPP, the loss is almost zero. The comparison between modified IC and IPSO is shown in Figure 9. For the initial MPP tracking, IPSO requires $200 \mathrm{~ms}$. In the case of retracking (after each step change in irradiance), IPSO requires approximately $40 \mathrm{~ms}$ to settle to a new MPP value, compared to modified IC that needs $60 \mathrm{~ms}$. The most probable reasons for the faster response of modified IC is the larger step size due to the particle flight.

In a typical sunny day, both the irradiance and temperature increase as the hour approaches the midday and thereafter decreases towards the evening. To study the performance of the MPPT algorithms under these gradual changes, such environmental scenario is simulated over a period of time. Figure 10 presents the irradiance and temperature profile, in which the irradiance and temperature are increased or decreased within one second rise or fall time, respectively.

A sequence of very fast irradiance variables was depicted in Figure 9. For incremental step, the power response time is equal to $43 \mathrm{~s}$ for modified IC algorithm for $95 \%$ in this case is shown in Figure 10 and $32 \mathrm{~s}$ for IPSO algorithm. Therefore, this shows that for the rapid irradiance variation, the IPSO responded very fast compared to the modified incremental conductance method. Nonetheless, the distinction among the maximized powers between these algorithms is also not negligible as depicted in Figure 10. If it is based on the final value, the relative error obtained between these two powers is about $4.8 \%$. The algorithm may be better than one another especially depending on the rate and speed of

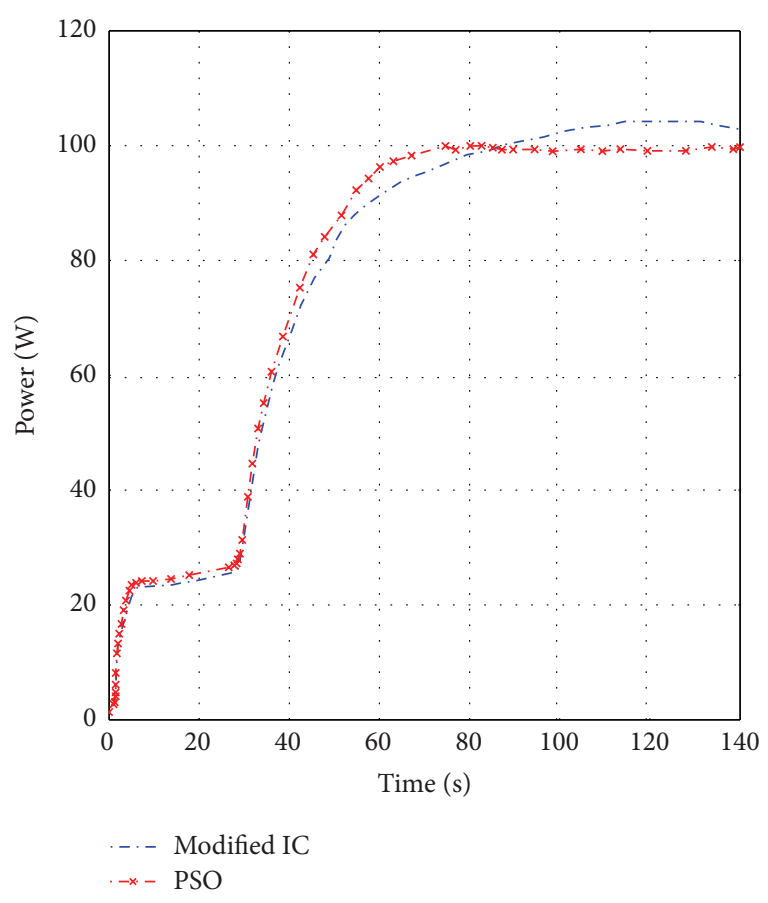

FIGURE 10: Comparison of proposed MPPT and conventional IC for daily Malaysian profile.

solar irradiance change, but without necessarily also having a good response time [19]. The difference becomes negligible for a significant time in terms of energy. It is well known that a photovoltaic system becomes interesting for energy production that requires a significant time.

Many researchers in the literature show that PSO algorithm is more inferior to other methods [19]. In this simulation, concurrently implementing the two algorithms in same given set of conditions proves that the IPSO-based MPPT has better efficiencies compared to modified IC, and it is easier to implement. The performance of IPSO method is found to be excellent compared to modified IC especially in terms of tracking speed and steady state oscillations. Though, in IPSO, the calculation of new duty cycles using (4) and (5) is simpler and does not take much time, and the number of sensors required will be less when experimenting. However, all two algorithms can be easily developed with the help of the low cost microcontroller.

\section{Performance Evaluation and Comparison}

Simulations evaluation of the proposed maximum power point tracking techniques under varying insolation shows that the photovoltaic module output power varies as in Figure 7(b). For the purpose of comparison, a modified incremental conductance method and $\mathrm{P} \& \mathrm{O}$ method was employed to track the MPP and the tracking performance of the IPSO-based MPPT method approach as shown in Figure 8. It is noticed that the maximum power reached by the photovoltaic system while using proposed IPSO-based MPPT algorithm is more efficient than the incremental conductance 
method. For the practical judgment of the effectiveness of the maximum power point tracking techniques, a variety insolation is assumed, and the photovoltaic power response is monitored for proposed method and compared with the work reported in [15] and the result was found to be as shown in Figure 10. It is now clear that the tracking steadfastness and speed of proposed maximum power point tracking technique are better than that of incremental conductance; also the tracking accuracy of the proposed techniques is superior to that of the incremental conductance.

\section{Conclusion}

In this paper, an IPSO-based MPPT method for tracking MPP either in unshaded or shaded irradiance levels was presented. An IPSO-based MPPT model utilizing a boost converter topology has also been presented. In order to speed up the searching technique, a learning factor and inertia weight were adapted. Linear decreases with increasing iteration numbers were adopted in this study for the weighting factor of the PSO formulas. The physical meaning of this modified weighting formula is that greater step sizes are used to increase the particle search velocity during the initial search because the distance to the global optimum is relatively large. This prevents an excessively small step size from making local optimum traps unavoidable. It has been demonstrated that an improved particle swarm optimization based MPPT method for tracking MPP is highly robust to variations in the solar insolation. The proposed control scheme is verified using Simulink models. The simulation results indicate that the converter can track the maximum power point of the photovoltaic system. The obtained results also confirmed that the convergence speed of the proposed method is high and the structure of the improved MPPT algorithms is so simple. With these results, the control scheme can be utilized for reliable and high quality photovoltaic system.

\section{Conflict of Interests}

The authors declare that there is no conflict of interests regarding the publication of this paper.

\section{Acknowledgments}

The authors would like to thank the Ministry of Higher Education, MOHE, Malaysia, for the financial support and Universiti Teknologi Malaysia, UTM JB, for providing the facilities to conduct the research.

\section{References}

[1] Y. Zou, Y. Yu, Y. Zhang, and J. Lu, "MPPT control for PV generation system based on an improved IncCond algorithm," Procedia Engineering, vol. 29, pp. 105-109, 2012.

[2] P. Bhatnagar and R. K. Nema, "Maximum power point tracking control techniques: state-of-the-art in photovoltaic applications," Renewable and Sustainable Energy Reviews, vol. 23, pp. 224-241, 2013.
[3] M. Abdulkadir, A. S. Samosir, and A. H. M. Yatim, "Modelling and simulation of maximum power point tracking of photovoltaic system in Simulink model," in Proceedings of the IEEE International Conference on Power and Energy (PECon '12), pp. 325-330, December 2012.

[4] M. A. Eltawil and Z. Zhao, "MPPT techniques for photovoltaic applications," Renewable and Sustainable Energy Reviews, vol. 25, pp. 793-813, 2013.

[5] H.-T. Yau, C.-J. Lin, and Q.-C. Liang, "PSO based PI controller design for a solar charger system," The Scientific World Journal, vol. 2013, Article ID 815280, 13 pages, 2013.

[6] K.-H. Chao, L.-Y. Chang, and H.-C. Liu, "Maximum power point tracking method based on modified particle swarm optimization for photovoltaic systems," International Journal of Photoenergy, vol. 2013, Article ID 583163, 6 pages, 2013.

[7] K.-H. Chao and Y.-H. Lee, "A maximum power point tracker with automatic step size tuning scheme for photovoltaic systems," International Journal of Photoenergy, vol. 2012, Article ID 176341, 10 pages, 2012.

[8] K.-H. Tang, K.-H. Chao, Y.-W. Chao, and J.-P. Chen, "Design and implementation of a simulator for photovoltaic modules," International Journal of Photoenergy, vol. 2012, Article ID 368931, 6 pages, 2012.

[9] K. Ding, X. Bian, H. Liu, and T. Peng, "A MATLAB-simulinkbased PV module model and its application under conditions of nonuniform irradiance," IEEE Transactions on Energy Conversion, vol. 27, no. 4, pp. 864-872, 2012.

[10] K. Ishaque, Z. Salam, A. Shamsudin, and M. Amjad, "A direct control based maximum power point tracking method for photovoltaic system under partial shading conditions using particle swarm optimization algorithm," Applied Energy, vol. 99, pp. 414-422, 2012.

[11] I. Houssamo, F. Locment, and M. Sechilariu, "Maximum power tracking for photovoltaic power system: development and experimental comparison of two algorithms," Renewable Energy, vol. 35, no. 10, pp. 2381-2387, 2010.

[12] X. Chen and Y. Li, "A modified PSO structure resulting in high exploration ability with convergence guaranteed," IEEE Transactions on Systems, Man, and Cybernetics, Part B, vol. 37, no. 5, pp. 1271-1289, 2007.

[13] K. Ishaque, Z. Salam, M. Amjad, and S. Mekhilef, "An improved particle swarm optimization (PSO)-based MPPT for PV with reduced steady-state oscillation," IEEE Transactions on Power Electronics, vol. 27, no. 8, pp. 3627-3638, 2012.

[14] Y.-H. Liu, S.-C. Huang, J.-W. Huang, and W.-C. Liang, "A particle swarm optimization-based maximum power point tracking algorithm for PV systems operating under partially shaded conditions," IEEE Transactions on Energy Conversion, vol. 27, no. 4, pp. 1027-1035, 2012.

[15] M. Abdulkadir, A. S. Samosir, A. H. M. Yatim, and S. T. Yusuf, "A new approach of modelling, simulation of mppt forphotovoltaic system in simulink model," ARPN Journal of Engineering and Applied Sciences, vol. 8, no. 7, pp. 488-494, 2013.

[16] M. Abdulkadir, A. S. Samosir, and A. H. M. Yatim, "Modeling and simulation of a solar photovoltaic system, its dynamics and transient characteristics in LABVIEW," International Journal of Power Electronics and Drive System, vol. 3, no. 2, pp. 185-192, 2013.

[17] R. W. Erickson and D. Maksimovic, Fundamentals of Power Electronics, Springer, 2001.

[18] H. Heydari-doostabad, R. Keypour, M. R. Khalghani, and M. H. Khooban, "A new approach in MPPT for photovoltaic array 
based on extremum seeking control under uniform and nonuniform irradiances," Solar Energy, vol. 94, pp. 28-36, 2013.

[19] M. A. Abdullah, A. H. M. Yatim, and C. W. Tan, "A study of maximum power point tracking algorithms for wind energy system," in Proceedings of the IEEE 1st Conference on Clean Energy and Technology (CET '11), pp. 321-326, June 2011.

[20] C.-M. Hong, T.-C. Ou, and K.-H. Lu, "Development of intelligent MPPT (maximum power point tracking) control for a gridconnected hybrid power generation system," Energy, vol. 50, no. 1, pp. 270-279, 2013. 

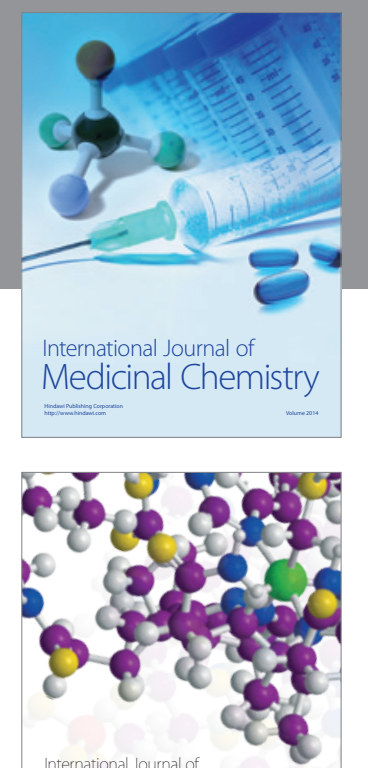

\section{Carbohydrate} Chemistry

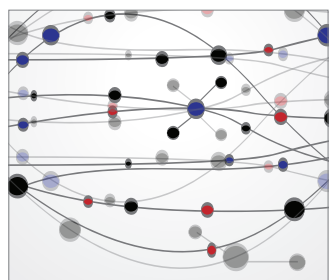

The Scientific World Journal
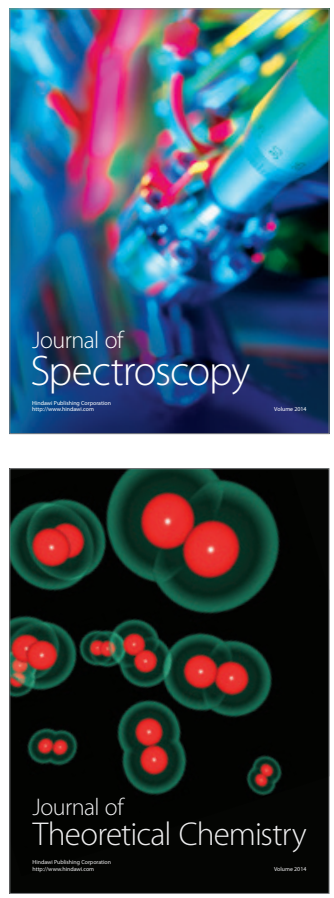
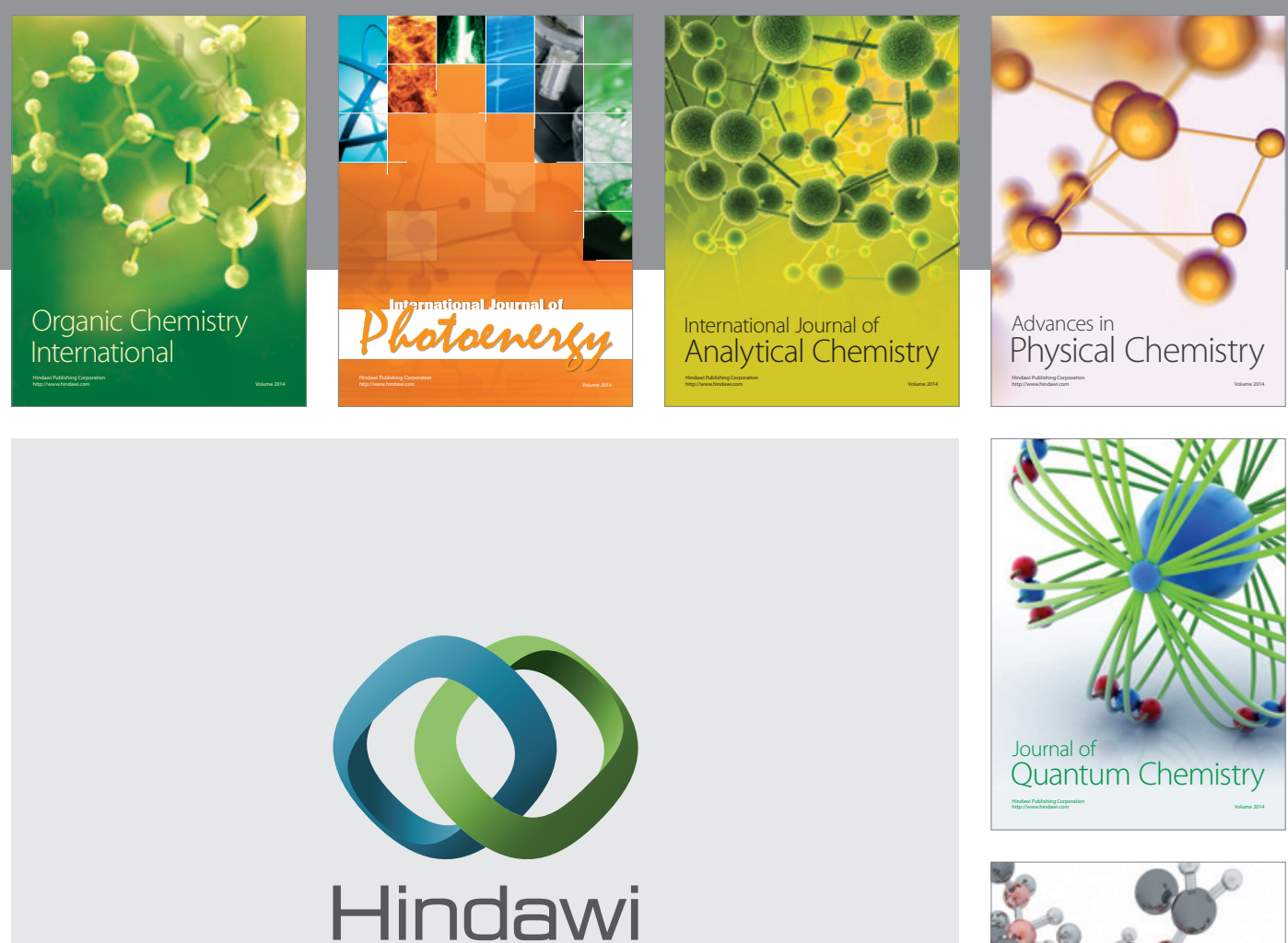

Submit your manuscripts at

http://www.hindawi.com

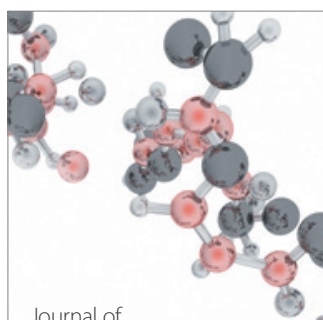

Analytical Methods

in Chemistry

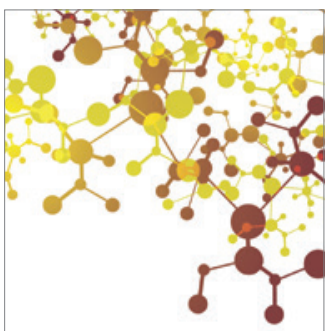

Journal of

Applied Chemistry

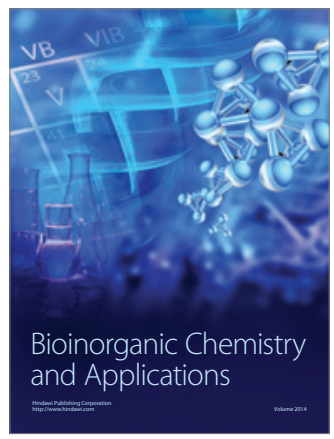

Inorganic Chemistry
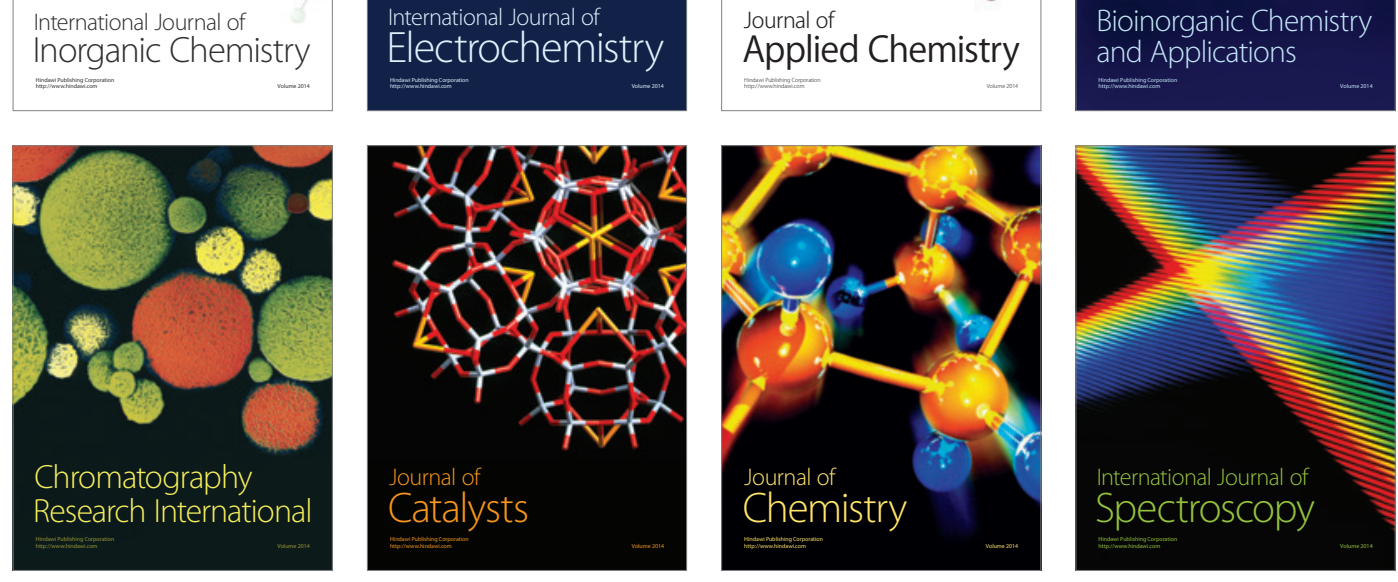